\title{
Flat band analogues and flux driven extended electronic states in a class of geometrically frustrated fractal networks
}

\author{
Atanu Nandy 6 Biplab Pal $\theta^{\circ}$ and Arunava Chakrabart \\ Department of Physics, University of Kalyani, Kalyani, West Bengal-741235, India
}

\begin{abstract}
We demonstrate, by explicit construction, that a single band tight binding Hamiltonian defined on a class of deterministic fractals of the $b=3 N$ Sierpinski type can give rise to an infinity of dispersionless, flat-band like states which can be worked out analytically using the scale invariance of the underlying lattice. The states are localized over clusters of increasing sizes, displaying the existence of a multitude of localization areas. The onset of localization can, in principle, be 'delayed' in space by an appropriate choice of the energy of the electron. A uniform magnetic field threading the elementary plaquettes of the network is shown to destroy this staggered localization and generate absolutely continuous sub-bands in the energy spectrum of these non-translationally invariant networks.
\end{abstract}

PACS numbers: 71.23.An, 72.15.Rn, 73.20.Hb, 73.23.Ad

\section{INTRODUCTION}

The interplay of geometry and quantum interference leading to exotic band structure in a class of quasi one-dimensional geometrically frustrated lattices (GFL) has drawn considerable interest in recent times. The GFL have been quite exhaustively studied over the years mainly in the context of localized spin systems [1], using the Heisenberg model for studying frustrated antiferromagnetism [2]- 7], or itinerant ferromagnetism within the Hubbard model [8, 9]. The degeneracy induced by the geometric frustration and non-trivial ground states such as the spin liquid states as its consequence [1] have unveiled rich physics of the spin systems.

Parallely, electronic spectra of several simple lattices, studied mainly within a single band tight binding formalism and only with nearest neighbor hopping, have displayed exotic features such as the appearance of dispersionless, flat bands (FB) [10]-14]. Such frustrated hopping models are important as they offer prospects of strong interaction physics such as the fractional quantum Hall effect. Graphene anti-dot lattices [15] and postgraphene materials such as the phosphorene [16] have been proposed as systems exhibiting the qualities for quantum computation [17] and an electrical tunability of quasi-flat bands respectively. Recent study on ultracold fermionic atoms patterned into finite two dimensional optical lattices of a kagomé geometry has added to such excitements [18]. Advancement in controlled growth of artificial, tailor-made lattices having atoms trapped in optical lattices with the complications of even a kagomé structure [19, 20] has contributed towards increasing interest in these deceptively simple looking systems.

The non-dispersive character of the energy $(E)$-wave vector $(k)$ curve makes the effective mass of the elec-

\footnotetext{
*Electronic address: atanunandy1989@gmail.com

${ }^{\dagger}$ Electronic address: biplabpal2008@gmail.com

‡Electronic address: arunava chakrabarti@yahoo.co.in
}

tron infinite, making the electron having such an energy practically immobile in the lattice. The corresponding single particle state is sharply localized at a point, or in a finite cluster of points in the lattice. Such clusters are separated from the neighboring clusters of the lattice by vertices where the amplitude of the wave function is zero. The energy eigenvalue corresponding to such a construction belongs to a flat band. Fig. 1 displays one such case, where the central hexagonal cluster has nonzero amplitude at the vertices, while the subset of atomic sites residing at the vertices of the triangle are zero. This figure corresponds to energy $E=-2$ in a tight binding description where the 'on-site' potential at every lattice point has been set equal to zero, and the nearest neighbor hopping integral has been assigned the value unity. Such situations can also be realized in the kagomé lattice [21], or other FB systems [22].

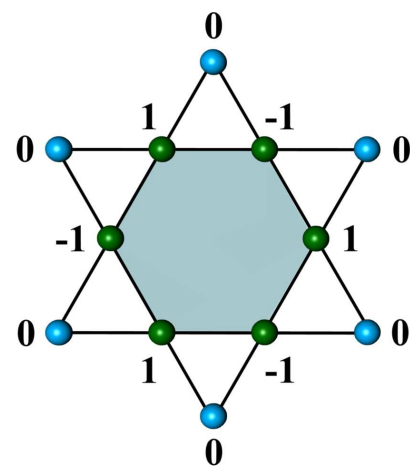

FIG. 1: (Color online) Schematic view of a part of an elementary star-network illustrating the localized distribution of amplitudes within a finite cluster.

The FB systems have been exemplified so far in terms of periodic lattice models. The number of flat bands in these systems is always finite. Comparatively speaking, practically nothing is known about the existence of dispersionless, flat band modes in hierarchically generated artificial lattices, where, because of the inherent scale in- 
variance, it might be possible to identify an infinity of such modes.

Here we address this issue in terms of a class of Sierpinski gasket (SPG) lattices, which have been widely studied over the years 23]-26] in respect of their electronic properties. As one should appreciate, the FB states are localized states with finite support in a lattice. Though, Anderson localization with weak disorder has been addressed for tight binding models with flat bands [27], the effect of self similar lattice topology in this context is something which we find practically unaddressed.

This leads to another, and a very important additional motivation for the present work, namely, an exact evaluation of at least a subsection of localized eigenstates in a hierarchical lattice. As such lattices are not periodic, one generally has to resort to an exact diagonalization of the Hamiltonian to know the energy eigenvalues, and because of the fragmented, Cantor set-like energy spectrum usually offered by these lattices 23] the eigenvalues obtained from one finite generation of the lattice in general, slip out of the spectrum on increasing the size of the system. A precise knowledge of the energy spectrum in the thermodynamic limit thus turns out to be a non trivial problem. Though in recent times the existence of an infinity of extended, fully transmitting eigenstates have been proven within the framework of real space renormalization group (RSRG) schemes [26, 28], the exact evaluation of localized state-eigenvalues has still largely remained an unresolved issue, until very recently [30, 31] and, for a special kind of deterministic fractal geometries.

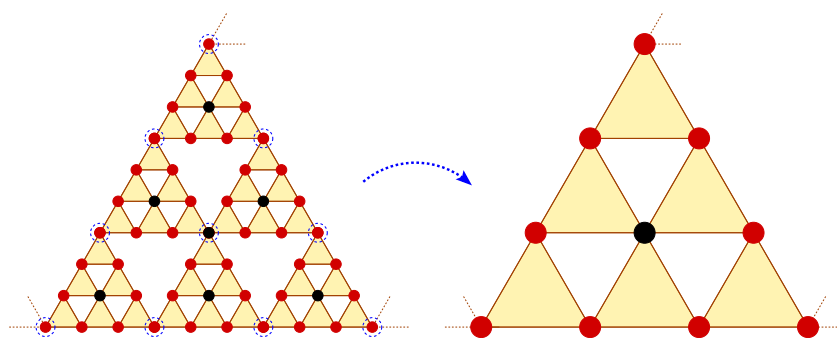

FIG. 2: (Color online) Portion of an infinite $b=3$ Sierpinski gasket (left) and its renormalized version. The circles colored red and black have coordination numbers four and six respectively. The encircled vertices survive after decimation, leading to the scaled lattice (right).

In this communication we focus on the so called $b=3 N$ Sierpinski gasket family [32]. We show that, it is possible to explicitly 'construct' localized eigenstates spanning finite sized clusters on such lattices. The clusters supporting such localized distribution of amplitudes of the wave function are separated from neighboring clusters by vertices where the amplitude of the wavefunction is zero at the specified energies. As a result, the electron with such energies are forced to hop around and remain confined in these finite clusters. The minimum size of the clusters supporting these localized states increases with the value of $n$. Using an RSRG scheme it is possible to unravel an infinity of such localized states and the corresponding spanning clusters for every member of the $b=3 N$ family. We present explicit results for the $b=3$ gasket. The remaining members can be easily dealt with using the same formalism, but with increasingly cumbersome algebra.

In the second phase of our work we examine the effect of a perturbation on the FB states in such hierarchical networks. The perturbation is chosen in the form of a uniform magnetic field piercing each elementary plaquette in the $b=3$ gasket. The flux threading each plaquette produces remarkable changes in the energy spectrum, generating apparent absolute continuity in the energy spectrum. Such subbands are populated only by extended wavefunctions, as revealed by exhaustive numerical study based on the RSRG scheme. The fractal character of the energy spectrum is removed, at least locally, and the lattice becomes completely transparent to an incoming electron when the Fermi level is chosen to lie in such continuous subbands. The FB states apparently disappear (at least, they could not be explicitly be constructed any more).

In what follows we describe our findings. In section II we describe the Hamiltonian and the RSRG scheme. Section [III contains a discussion of the construction of the localized eigenstates on finite supports. A critical discussion on the flat band status of such states are provided in Section IV Section $\nabla$ highlights the effect of inserting a uniform magnetic flux in each elementary plaquette of the $b=3$ gasket, and the two-terminal transport calculations are discussed in Section VI, Finally, in section VII we draw our conclusions.

\section{THE HAMILTONIAN AND THE RSRG SCHEME}

We refer to Fig. 2 where a portion of a $b=3$ SPG network (left) and its renormalized version are shown (right). The electron's hopping is restricted only along each edge, and between nearest neighbors only. We distinguish between two types of sites, viz., one with coordination number four and colored in red (henceforth called $C_{4}$ sites) and the other, black circles, having a coordination number six $\left(C_{6}\right.$ sites). The system is described by the tight binding Hamiltonian,

$$
\boldsymbol{H}=\sum_{i} \epsilon_{i} c_{i}^{\dagger} c_{i}+\sum_{\langle i j\rangle} t_{i j}\left[c_{i}^{\dagger} c_{j}+\text { h.c. }\right]
$$

$\epsilon_{i}$ is the on-site potential at each vertex, and will be assigned symbols $\epsilon_{4}$ and $\epsilon_{6}$ for the $C_{4}$ and $C_{6}$ sites respectively. $t$ is the nearest neighbor hopping integral. We shall be interested only in the localization induced by the topology of the lattice, and hence, in all numerical results we shall set $\epsilon_{4}=\epsilon_{6}=0$, and $t=1$. However, on renormalization, the on-site potentials deviate from each 
other at every stage, and that is why it is necessary to distinguish them at the very beginning.

Using the set of difference equations satisfied by the electrons, viz.,

$$
\left(E-\epsilon_{i}\right) \psi_{i}=\sum_{j} t_{i j} \psi_{j}
$$

we obtain a scaled version of the original lattice, as shown in Fig. 2. An appropriate subset of the $C_{4}$ and $C_{6}$ sites are decimated to yield an identical geometry with the renormalized on-site potentials and the hopping integral. The 'surviving' sites on the renormalized lattice are encircled in the left portion of Fig. 2, The RSRG recursion relations for the on-site potentials and the hopping integrals are given by,

$$
\begin{aligned}
\epsilon_{6, n+1} & =\epsilon_{6, n}+3 \mathcal{F}_{n} \\
\epsilon_{4, n+1} & =\epsilon_{4, n}+2 \mathcal{F}_{n} \\
t_{n+1} & =\frac{\beta_{2, n} E^{2}+\gamma_{2, n} E+\delta_{2, n}}{\Delta_{n}}
\end{aligned}
$$

where, $\mathcal{F}_{n}=\left(\alpha_{1, n} E^{3}+\beta_{1, n} E^{2}+\gamma_{1, n} E+\delta_{1, n}\right) / \Delta_{n}$, and,

$$
\begin{aligned}
\alpha_{1, n}= & 2 t_{n}^{2} \\
\beta_{1, n}= & -2 t_{n}^{2}\left(t_{n}+2 \epsilon_{4, n}+\epsilon_{6, n}\right) \\
\gamma_{1, n}= & -2 t_{n}^{2}\left[5 t_{n}^{2}-t_{n}\left(\epsilon_{4, n}+\epsilon_{6, n}\right)-\right. \\
& \left.\epsilon_{4, n}\left(\epsilon_{4, n}+2 \epsilon_{6, n}\right)\right] \\
\delta_{1, n}= & -2 t_{n}^{2}\left[2 t_{n}^{3}+t_{n} \epsilon_{4, n} \epsilon_{6, n}+\epsilon_{4, n}^{2} \epsilon_{6, n}-\right. \\
& \left.t_{n}^{2}\left(4 \epsilon_{4, n}+\epsilon_{6, n}\right)\right]
\end{aligned}
$$

The other symbols used are,

$$
\begin{aligned}
& \beta_{2, n}=t_{n}^{3} \\
& \gamma_{2, n}=t_{n}^{3}\left(4 t_{n}-\epsilon_{4, n}-\epsilon_{6, n}\right) \\
& \delta_{2, n}=t_{n}^{3}\left(\epsilon_{4, n} \epsilon_{6, n}+2 t_{n}^{2}-4 t_{n} \epsilon_{4, n}\right) \\
& \Delta_{n}=\left[\left(E-\epsilon_{4, n}\right)^{2}-t_{n}^{2}\right]\left[\left(E-\epsilon_{6, n}\right)\left(E-\epsilon_{4, n}-2 t_{n}\right)-6 t_{n}^{2}\right]
\end{aligned}
$$

The 'stage' of renormalization is denoted by $n \geq 0$, and we have chosen to stick to $\epsilon_{4,0}=\epsilon_{6,0}=\epsilon$ and $t_{0}=t$ at the bare scale of length.

The recursion relations Eq. (3) will now be exploited to construct and analyze a class of localized and extended states in such systems.

\section{CONSTRUCTING THE LOCALIZED STATES}

\section{A. The basic scheme}

Let us begin with the $b=3$ gasket and refer to Fig. 3. It is simple to work out that, if we set $E=\epsilon_{4}-2 t$, a consistent solution of the difference equations (or equivalently, the Schrödinger equation) is obtained in which the amplitude of the wave function $\psi_{j}$ is zero at the $C_{6}$ vertices with coordination number six and lying at the center of the shaded hexagon, as well as on a subset of $C_{4}$ vertices. The non-zero amplitudes are designated by $x$ (which can be set equal to unity, for example). This is illustrated in the left panel of Fig. 3 This latter subset of $C_{4}$ sites sit at the 'connecting' points between hexagonal plaquettes at different portions of the infinite lattice. By making the amplitude vanish at these strategic sites it is possible to confine the hopping of the electron around the periphery of the shaded hexagons. The eigenstate resembles the spirit of a molecular state 29] localized partially by quantum interference and partially by physical boundary formed by the sites with zero amplitude. The wave function corresponding to this energy eigenvalue does not have any overlap with the wave function described on the next neighboring hexagons. The localization area is the shaded hexagonal plaquette confining the electron's motion for $E=\epsilon_{4}-2 t$ along the 'periphery', and is distributed throughout the infinite geometry.

\section{B. Exploiting the self similarity}

Once such a construction of localized state-amplitudes is made possible in the bare length scale, the self similar structure of the SPG lattices can be exploited to construct wave functions, and to 'work out' the corresponding areas of localization at various scales of length. The eigenvalues corresponding to such cases are easily extracted from the solutions of the equation

$$
E=\epsilon_{4, n}-2 t_{n}
$$

at any desired $n$-th stage of RSRG. For every real root of this equation, a construction similar to that in the original lattice can be made in respect of the values of the amplitudes of the wave function at different $C_{4}$ and $C_{6}$ vertices on the scaled version of the lattice. One then has to work in the reverse direction to lay out the amplitudedistribution on the original lattice at the bare scale of length by simultaneously solving the difference equations Eq. (2) for the respective energy eigenvalue. The scheme is illustrated in Fig. 3(b). With $\epsilon_{4}=\epsilon_{6}=0$ and $t=1$, we get $E=-2$ at the original length scale, while the same parameters yield localized states at $E=-2$ and $E=1 \pm \sqrt{2}$ when we set $n=1$, that is, when we solve the equation $E=\epsilon_{4,1}-2 t_{1}$. Let us now explain the construction of localized states for the new roots, viz., $E=1 \pm \sqrt{2}$.

The hexagon in Fig. 3(b) whose sides are highlighted by thick green line is the 'smallest' hexagon on a one step renormalized gasket. We choose the distribution of amplitudes at $E=\epsilon_{4,1}-2 t_{1}$ around this hexagon exactly in the same way as we did for $E=-2$ in the bare length scale (Fig. 3(a)). Such a construction, as we now appreciate, can be made consistently throughout the infinite system. The next job is to work out, in the reverse direction, the amplitudes of the wave function at all the ver- 


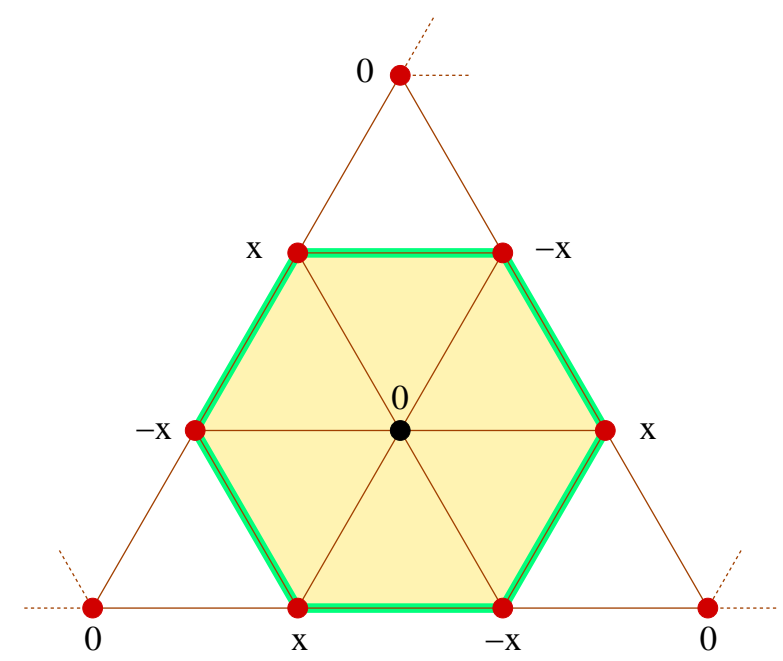

(a)

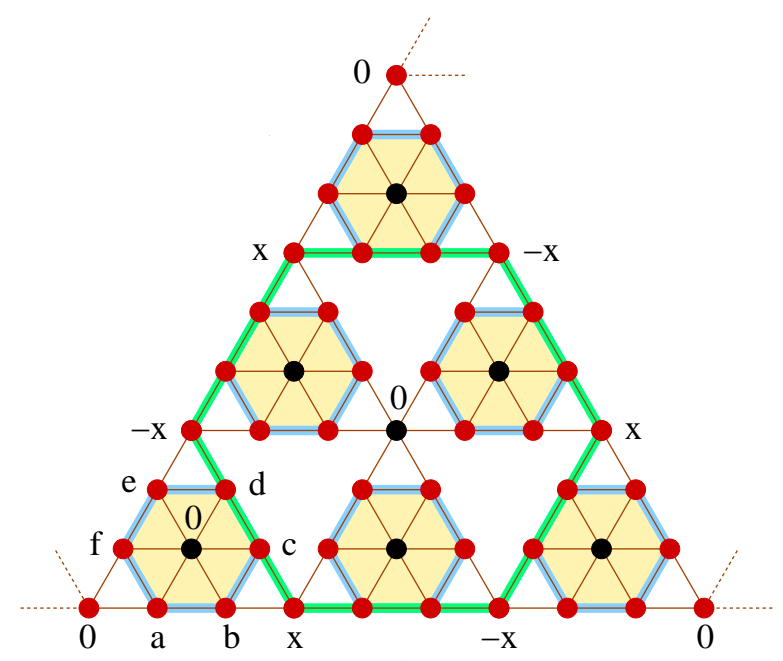

(b)

FIG. 3: (Color online) (a) Distribution of a flat-band state amplitudes on a $b=3$ SPG network at its bare scale of length, and (b) the distribution on a one step renormalized gasket. The localized state eigenvalues are extracted from the equations $E=\epsilon_{4,0}-2 t_{0}$ and $E=\epsilon_{4,1}-2 t_{1}$ respectively.

tices of the original gasket with the prefixed amplitudes (with values $\pm x$ at the edges of the green highlighted hexagon and zero at its center) for energy eigenvalues $E=1 \pm \sqrt{2}$.

The amplitudes are denoted by the letters $a-f$ in Fig. 3(b). We have shown explicitly the smaller hexagons (blue highlighted) that are embedded in the larger one (green highlighted). It is possible to obtain a consistent set of values for the amplitudes at all vertices of the smaller (blue) hexagons. For example, with $x=1$, set arbitrarily, the distribution of amplitudes for $E=1+\sqrt{2}$ (one solution of $E=\epsilon_{4,1}-2 t_{1}$ with $\epsilon=0$ and $t=1$ ) is given by, $a=-f=(\sqrt{2}-1) / 2, b=-e=1 / \sqrt{2}$ and $c=-d=1 / 2$. The amplitude at the center of a small hexagon is zero for this particular distribution. An identical pattern of distribution, but with a different set of values for $a, b, \ldots f$, can be easily obtained if one sets $E=1-\sqrt{2}$.

Needless to say, the scheme can be extrapolated to unravel other localized states by solving the equation $E=\epsilon_{4, n}-2 t_{n}$ at any desired level. The roots increase in number, and the set of solutions obtained at the $n$-th stage of renormalization contains all the roots obtained from the $(n-1)$-th stage. In every case one has to identify the smallest hexagon in that scale of length, set the amplitude of the wave function equal to zero at its center. The sites decorating the periphery of the basic hexagon then should be assigned amplitudes equal to $\pm x$ as we did in Fig. 3. Such a basic hexagon at the renormalized scale must be separated from its neighboring hexagons, a condition that is easily satisfied by setting the amplitude at the 'connecting' sites equal to zero. The final task is to map the distribution back onto the original lattice and to solve the set of equations Eq. (2) to work out the amplitudes at the inner sites of the lattice at its bare scale of length.

\section{The 'staggered' character of localization}

An interesting qualitative distinction between the energy eigenvalues corresponding to these localized states can be unravelled if we look at the evolution of the hopping integral under RSRG steps. For any energy eigenvalue obtained from the $n$-th stage, and by solving the equation $E=\epsilon_{4, n}-2 t_{n}$, the hopping integral remains finite upto $n$-th RSRG steps. This means that one has non-zero overlap of the wave function between nearest neighboring sites at that length scale. When mapped back onto the original lattice it amounts to a non-zero overlap of the wave function between sites much beyond the nearest neighbors. However, the hopping integral starts decaying after the $n$-th RSRG step implying that the wave function looks 'extended' only locally, and in reality such wave functions are localized on a finite cluster of the infinite lattice. The overlap of the envelopes of the wave function for a localized state-energy extracted at the $n$-th stage weakens beyond a certain scale of length. Such finite sized clusters span larger and larger areas in real space as one extracts the energy eigenvalue by solving the above equation at deeper and deeper scales of length.Thus simply by 'choosing' the value of stage of renormalization ' $n$ ' one can delay, in space, the localizing character of the wave function. In a recent study [30] we encountered such staggered localization in a special class of fractal lattices.

Before we end this section, it is pertinent to point out that the size of the spanning clusters even at the bare length scale depends on the value of $n$ in the class of $b=3 N$ SPG fractals. Fig. [ depicts three other cases, 


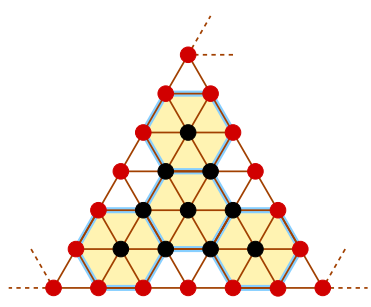

(a)

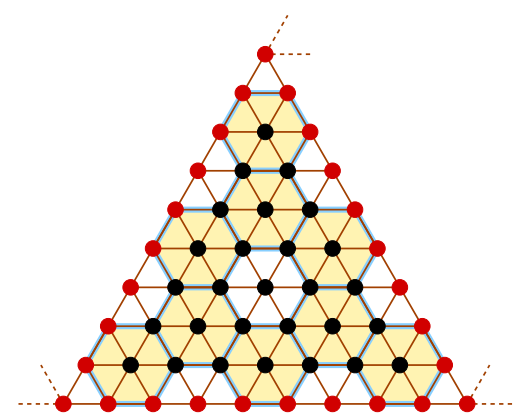

(b)

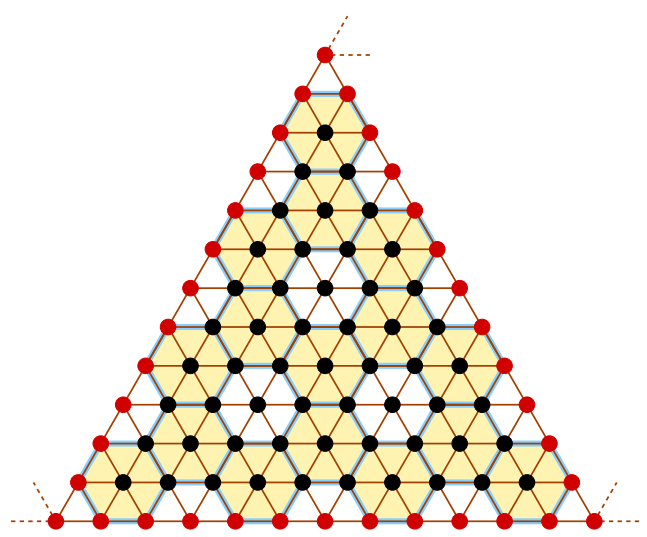

(c)

FIG. 4: (Color online) Distribution of amplitudes for a non-dispersive eigenstate at $E=\epsilon_{4}-2 t$ on $b=6, b=9$ and $b=12$ Sierpinski gaskets. With increasing scale factor, the amplitudes assume non-trivial distribution over larger and larger clusters which ultimately get decoupled from the rest of the lattice, courtesy, the zero amplitudes at the connecting vertices.

viz., $b=6, b=9$ and $b=12$. We stick to the same value of energy, viz., $E=\epsilon_{4}-2 t$. The localization areas even at the bare length scale are already larger than that in the $b=3$ case. In each case, the electron's hopping is confined around the peripheries of clusters of finite size, shown by the shaded areas. Such finite sized clusters grow bigger and bigger as one goes from $b=6$ to $b=12$, and beyond. The clusters, however big, are ultimately separated from each other by a subset of $C_{4}$ sites with $\psi_{j}=0$, and are distributed throughout the infinite geometry.

\section{THE FLAT BAND ANALOGUES}

A pertinent question is, whether these infinite number of localized eigenstates are non-dispersive in character, in the spirit of the flat band states observed in a class of periodic lattices as mentioned in the introduction. The absence of translational invariance in the SPG class of lattices prevents a derivation of the usual energy-wave vector dispersion relation. At the same time, the fact that the localized states discussed so far have been 'constructed' so as to display a kind of self localization, where the amplitudes are distributed over a finite cluster of lattice points, effectively 'decoupled' from the rest of the system. This inspires us to undertake a couple of indirect, but effective ways of discerning the actual character of these states.

\section{A. Dispersion relation for a periodic array of fractal approximants}

First, we construct a periodic array of $b=3$ SPG networks. Each 'unit cell' consists of a finite SPG at its $n$-th generation. One such array comprising the first generation $b=3$ SPG network is shown in Fig. 5 for illustration.
As is evident, such a construction requires the presence of a top vertex with coordination number two (colored cyan in Fig. 5). It is important to appreciate that for such finite systems the distribution of amplitudes does not follow the method prescribed for Fig. 3, which was typical of an infinite system where the 'end points' were not 'visible'.

As we gradually increase the value of $n$, the unit cell itself turns out to be a reasonably good approximant of the infinite system, and becomes the true representative in the limit $n \rightarrow \infty$. For a periodic array of $n$-th generation SPG networks one can renormalize each 'unit cell' to $n$-times to convert the array into an array of triangles with the potentials at the vertices equal to $C_{2, n}$ and $C_{4, n}$ respectively. The nearest neighbor hopping integral along the edges of such effective triangles are $t_{n}$. This array of triangles is then converted into an array of single 'effective' atomic sites (encircled by dotted lines in Fig. 5) with energy dependent potential, and inter-connected by an energy dependent hopping integral. The effective, energy dependent on-site potential and the nearest neighbor hopping integral in the linear chain of renormalized atoms are given by,

$$
\begin{aligned}
& \tilde{\epsilon}=\epsilon_{4, n}+\frac{2 t_{n}^{2}}{E-\epsilon_{2, n}} \\
& \tilde{t}=\frac{t_{n}^{2}}{E-\epsilon_{2, n}}
\end{aligned}
$$

In this case, we need an additional renormalization of the top vertex with coordination number two, viz.,

$$
\epsilon_{2, n+1}=\epsilon_{2, n}+\mathcal{F}_{n}
$$

The definition of $\mathcal{F}_{n}$ is already given in earlier section (Eq.(41) and Eq.(5) ). The $E$ - $k$ dispersion relation for such a periodic chain is then easily obtained through the equation,

$$
E=\tilde{\epsilon}+2 \tilde{t} \cos k a
$$




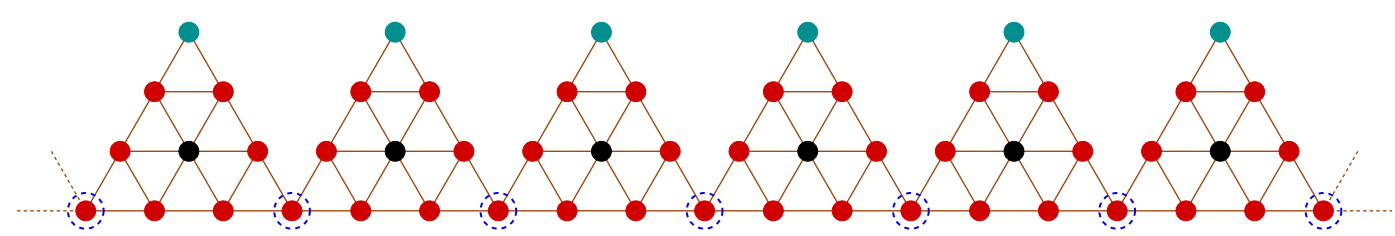

FIG. 5: (Color online) A periodic array of finite generation $b=3$ SPG networks. With increasing generation each unit cell will be representative of an infinite SPG network.

In Fig. 6] we depict the dispersion relation when the
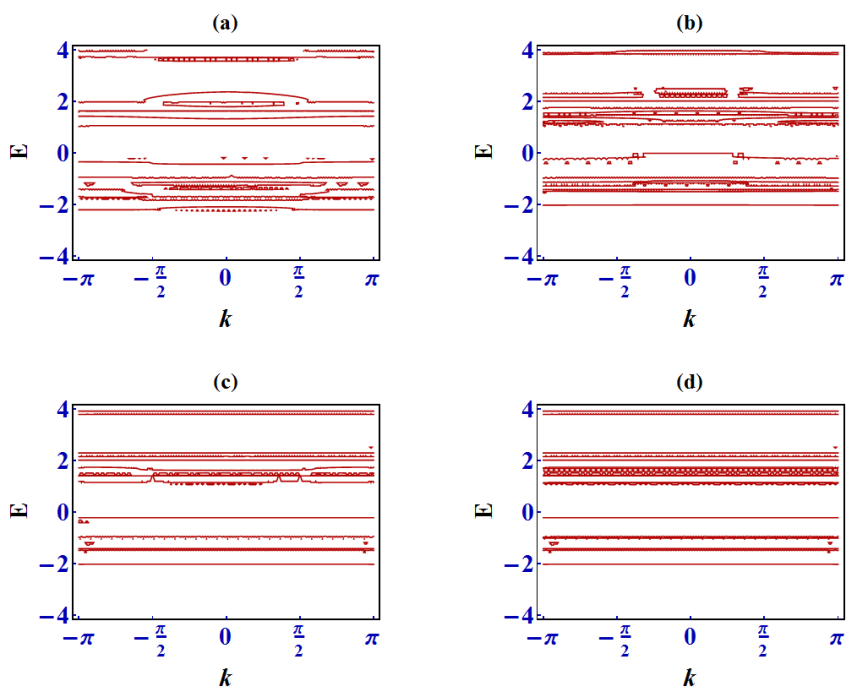

FIG. 6: (Color online) The dispersion relation for the array depicted in Fig. 5] The flat, dispersionless modes are apparent. The flat band at $E=-2$, the root of the equation $E=\epsilon_{4}-2 t$ with $\epsilon_{4}=\epsilon_{6}=0$ and $t=1$ has already been shown to exist, by explicit construction, for an infinite system.

unit cell consists of (a) the first generation, (b) the second generation, (c) the third generation and (d) the fourth generation $b=3 \mathrm{SPG}$ networks. We have set $\epsilon_{4}=\epsilon_{6}=0$ and $t=1$. It is clearly seen form Fig. [6(c) and (d) that flat, non-dispersive bands appear at $E=-2$ and at $E=1 \pm \sqrt{2}$ - the three roots obtained from the equation $E=\epsilon_{4, n}-2 t_{n}$ at the bare length scale and from the one step renormalized lattice, as discussed in the last section. Even with a modest unit cell structure for the triangular array, the self (staggered) localized states corresponding to the truly infinite system are showing up. Thus, we feel tempted to identify these self-localized states as flat band analogues of similar states in quasi one dimensional systems [10]- 13]. However, we are now in a position to appreciate that such states will be infinite in number as the size of the system approaches the thermodynamic limit.

\section{B. A study of the density of states}

As a second check, we evaluate the local density of states (LDOS) at the $C_{4}$ and $C_{6}$ sites. The results are shown in Fig. 17(a) and (b) respectively. This is done following the standard procedure, and using the RSRG recursion relations Eq. (3), which finally yield the local Green's function (LGF) at the $C_{4}$ and $C_{6}$ sites as, $G_{00}^{(4)}=\left(E-\epsilon_{4}^{*}\right)^{-1}$ and $G_{00}^{(6)}=\left(E-\epsilon_{6}^{*}\right)^{-1}$ respectively. The symbol $\epsilon_{j}^{*}$ indicates the fixed point value of the respective parameter. Finally, the LDOS at the desired site is obtained as, $\rho_{j}=(-1 / \pi) \lim _{\eta \rightarrow 0} \operatorname{Im} G_{00}^{(j)}(E+i \eta)$, with $j=4$ or 6 depending on the coordination number.

The LDOS (in the absence of an external magnetic field) exhibits a highly fragmented distribution as seen in Fig. 7(a) and (b). This is expected. The fine structure is related to the distribution of spectral weight [33], and is controlled by the lattice topology. One should observe that the $j$-th site $\left(C_{4}\right.$ or $\left.C_{6}\right)$ having $\psi_{j}=0$ can not contribute to the LDOS at that particular site. Nevertheless, non-trivial contribution comes from the neighboring sites yielding a finite value of the density of states at a $C_{4}$ site.

It is important to appreciate the role of the numerical value of the imaginary part $\eta$ added to the energy. $\eta$ determines the width of the LDOS around an energy. We have carefully examined the growth of the LDOS for $E=-2,1 \pm \sqrt{2}$ and at several other localized flat band states as obtained by explicit construction at bare length scale and from a one-step renormalized lattice. The result is tabulated in Table \for the above three energy eigenvalues. It is explicitly seen that, for each of these energy eigenvalues, with a sequential decrease in the value of $\eta$, the LDOS at the $C_{4}$ site, viz., $\rho_{4}$ increases exponentially, indicating that in the limit $\eta \rightarrow 0$, the LDOS diverges at these special energy values. As we know, the average density of states (ADOS) is related to the group velocity $v_{g}$ of the electron by the relation

$$
\rho_{a v} \propto \int v_{g}^{-1} d^{3} k
$$

where, $v_{g} \propto d E / d k$. Therefore, a diverging LDOS, leading to a diverging ADOS, is indicative of the zero (or extremely low) group velocity and hence, a non-dispersive character of the state. The energy eigenvalues, obtained by construction, and corresponding to the self-localized eigenfunctions in the present example can thus be considered as the so called flat band states. We can thus unravel infinite number of such non-dispersive modes in an infinite $b=3 N$ SPG network. 


\begin{tabular}{|c|c|c|c|}
\hline$E$ & $\eta$ & $\rho_{4}(E)$ & $\rho_{6}(E)$ \\
\hline \multirow{7}{*}{-2} & $10^{-2}$ & 3.28 & 0.03 \\
\hline & $10^{-3}$ & 15.66 & 0.07 \\
\hline & $10^{-4}$ & 74.38 & $1.5 \times 10^{-3}$ \\
\hline & $10^{-5}$ & 355 & $3.2 \times 10^{-4}$ \\
\hline & $10^{-6}$ & 1703.2 & $6.8 \times 10^{-5}$ \\
\hline & $10^{-7}$ & 8701 & $1.34 \times 10^{-5}$ \\
\hline & $10^{-8}$ & 40419 & $2 \times 10^{-6}$ \\
\hline \multirow{7}{*}{$1-\sqrt{2}$} & $10^{-2}$ & 1.68 & 1.06 \\
\hline & $10^{-3}$ & 5.2 & 0.5 \\
\hline & $10^{-4}$ & 22.5 & 0.11 \\
\hline & $10^{-5}$ & 106.4 & $2 \times 10^{-2}$ \\
\hline & $10^{-6}$ & 506.24 & $5.4 \times 10^{-3}$ \\
\hline & $10^{-7}$ & 2402.8 & $1.13 \times 10^{-3}$ \\
\hline & $10^{-8}$ & 11454 & $2.4 \times 10^{-4}$ \\
\hline \multirow{7}{*}{$1+\sqrt{2}$} & $10^{-2}$ & 1 & $7.5 \times 10^{-2}$ \\
\hline & $10^{-3}$ & 4.41 & $1.75 \times 10^{-2}$ \\
\hline & $10^{-4}$ & 20.91 & $3.8 \times 10^{-3}$ \\
\hline & $10^{-5}$ & 100 & $8 \times 10^{-4}$ \\
\hline & $10^{-6}$ & 473 & $1.7 \times 10^{-4}$ \\
\hline & $10^{-7}$ & 2249 & $3.6 \times 10^{-5}$ \\
\hline & $10^{-8}$ & 10747 & $7.4 \times 10^{-6}$ \\
\hline
\end{tabular}

TABLE I: The values of the local densities of states $\rho_{4}(E)$ and $\rho_{6}(E)$ at special energy eigenvalues $E=-2$, and $1 \pm \sqrt{2}$ as obtained from the original lattice and its one-step renormalized version corresponding to localized flat-band states. We have set the on-site potential $\epsilon=0$ at each site, and the energy is measured in unit of the hopping integral $t$.

\section{EFFECT OF A UNIFORM MAGNETIC FIELD}

As is well known, the FBS are unstable against perturbation. We examine the general character of the energy spectrum of a $b=3 \mathrm{SPG}$ network when each elementary plaquette is threaded by a uniform magnetic flux $\Phi$. The presence of a magnetic field breaks the time reversal symmetry of the electron-hopping along a bond, and this is taken care of by introducing a Peierls' phase in the hopping integral, viz., $t \rightarrow t \exp \left(i 2 \pi \Phi / 3 \Phi_{0}\right)$, where $\Phi_{0}=h c / e$ is the fundamental flux quantum.

The RSRG method is used again to evaluate the LDOS, which is shown in Fig. 7(c) and (d) for the $C_{4}$ and $C_{6}$ sites respectively for $\Phi=\Phi_{0} / 4$. We draw the attention of the reader to the absolutely continuous shaded portions in the LDOS. This is a generic character for all values of the piercing flux. We have carefully scanned the continuous subbands in arbitrarily small energy intervals, and observed the flow of the hopping integral under the RSRG iterations. Extensive numerical search reveals that for any energy belonging to the continua, the hopping integral keeps on oscillating for an indefinite number of iteration loops. This implies that, for any energy forming the continua there is non-zero over- lap between the envelopes of the wave function at various scales of length, and that, the subbands are densely populated by extended wave functions. In addition, the energy $E=0$ lying at the center of the spectrum exhibits a one cycle fixed point in the parameter space, that is, $\left(\epsilon_{4, n}, \epsilon_{6, n},\left|t_{n}\right|\right)=\left(\epsilon_{4, n+1}, \epsilon_{6, n+1},\left|t_{n+1}\right|\right)$, for $n \geq 0$.
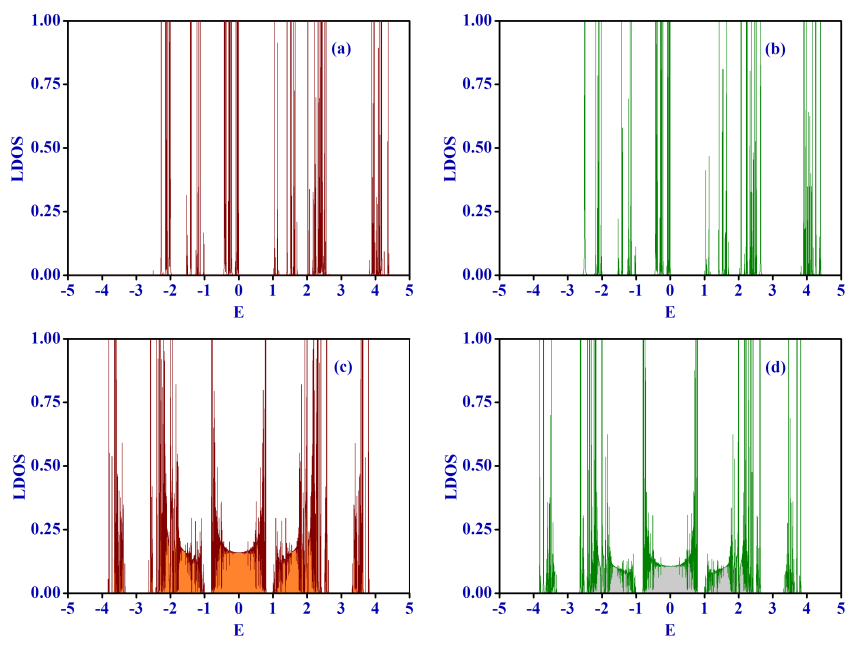

FIG. 7: (Color online) Local density of states at the (a) $C_{4}$ and (b) $C_{6}$ sites of an infinite $b=3$ SPG network in the absence of a magnetic field. (c) Local density of states at a $C_{4}$ site when each elementary plaquette is pierced by a magnetic flux $\Phi=\Phi_{0} / 4$, and (d) the same at a $C_{6}$ site with $\Phi=\Phi_{0} / 4$. We have set $\epsilon_{4}=\epsilon_{6}=0$ and $t=1$. The imaginary part added to the energy is $\eta=10^{-4}$.

The existence of the subbands of extended states does not rule out the other localized states, though the nondispersive, flat bands apparently do not exist (or, at least, become undetectable). As, for an electron circulating around a closed loop, the magnetic flux plays a role equivalent to the wave vector [34], we illustrate the $E-\Phi$ diagram (Fig. 8) as an analogue of the dispersion relation in Fig. 6. The energy eigenvalues of the fractal network are seen to form mini bands as a function of the flux $\Phi$ with period $\Phi_{0}$. There are multiple, inter-twined band crossings, and quite a dense distribution of eigenvalues, forming almost continuous quasi-flat $E-\Phi$ 'bands'. The pattern is flux periodic with a period equal to one flux quantum, and close observation of the $E-\Phi$ 'band' subsections reveals formation of interesting variants of the Hofstadter butterflies [35]. The spectral landscape is a quantum fractal, and encoding the gaps with appropriate topological quantum numbers remains an open problem for such deterministic fractals.

\section{TWO-TERMINAL TRANSMISSION CHARACTERISTICS}

For the sake of completeness of the above discussion, we have computed the two-terminal transmission characteristics of a finite system. The procedure is standard and 


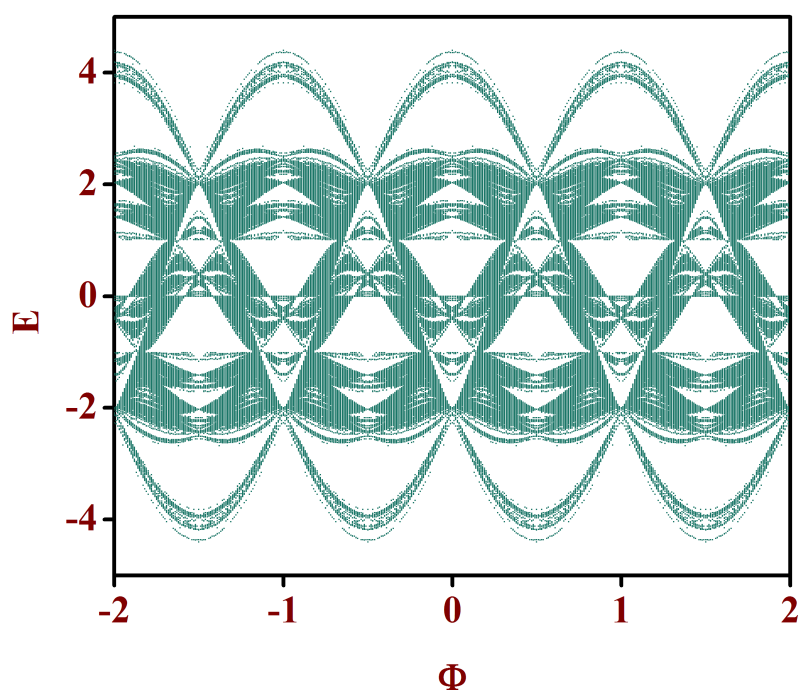

FIG. 8: (Color online) Distribution of energy eigenvalues against the magnetic flux $\Phi$ (in unit of $\Phi_{0}=h c / e$ ) in an infinite $b=3$ SPG network.

is often used to evaluate the transmission coefficient of such hierarchical fractal structures 30]. The basic idea is to clamp the system in between two semi-infinite periodic leads, the so called 'source' and the 'drain'. The on-site potential at the atomic sites in the leads is $\mathcal{U}_{L}$. The hopping integral is $\mathcal{T}_{L}$ in between the nearest neighboring atomic sites in the leads. The finite sized network sandwiched in between the two ordered leads is then successively renormalized to reduce it to an effective diatomic molecule (dimer) [30]. The transmission coefficient of the lead-network-lead system then is given by a well-known formula [36],

$$
\begin{gathered}
T=\frac{4 \sin ^{2} k a}{|\mathcal{A}|^{2}+|\mathcal{B}|^{2}} \\
\text { with, } \mathcal{A}=\left[\left(M_{12}-M_{21}\right)+\left(M_{11}-M_{22}\right) \cos k a\right] \\
\text { and } \quad \mathcal{B}=\left[\left(M_{11}+M_{22}\right) \sin k a\right]
\end{gathered}
$$

where, $M_{i j}$ refer to the dimer-matrix elements, written appropriately in terms of the on-site potentials of the final renormalized left $(\mathrm{L})$ and right $(\mathrm{R})$ atoms at the extremities of the finite SPG network and the renormalized hopping between them. $\cos k a=\left(E-\mathcal{U}_{L}\right) / 2 \mathcal{T}_{L}$, and $a$ is the lattice constant in the leads which is set equal to unity throughout the calculation.

It is apparent from Fig. 9 that, the low transmitting character of the gasket in the absence of an external magnetic field (Fig. 9(a)) is enriched by high transmitting windows in the presence of a magnetic flux (Fig. 9(b)). This corroborates the argument about the existence of absolutely continuous subbands populated by extended eigenfunctions.

The transmission coefficient, as expected, is flux periodic, with period equal to one flux quantum. The fine structures of the Aharonov-Bohm oscillations in transport are of course, sensitive to the chosen energy eigen-
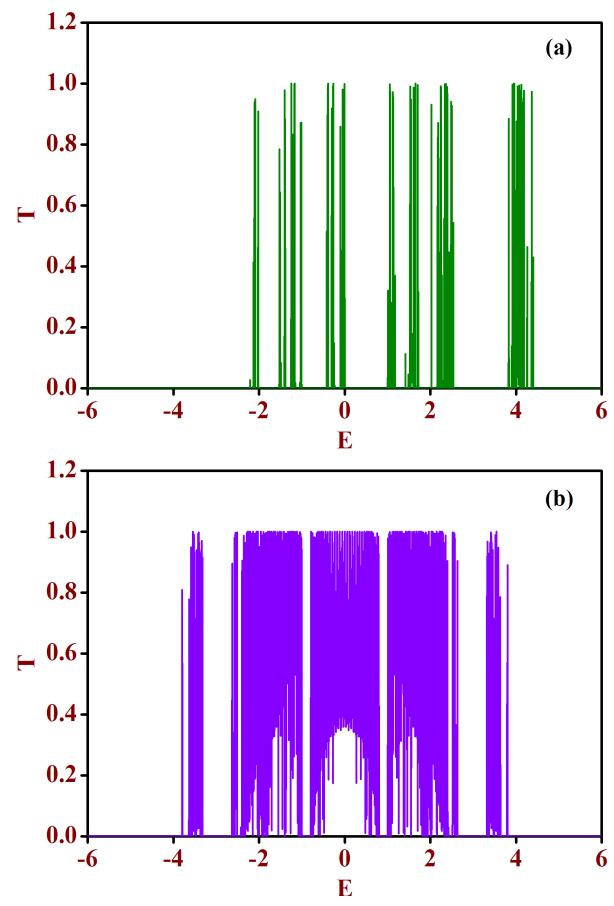

FIG. 9: (Color online) Transmission coefficient of a 4-th generation $b=3$ SPG network with (a) $\Phi=0$, and (b) $\Phi=\Phi_{0} / 4$. We have chosen $\epsilon_{4}=\epsilon_{6}=0, t=1, \mathcal{U}_{L}=0$ and $\mathcal{T}_{L}=3$.

value. However, to save space, we refrain from showing these figures.

\section{CONCLUSION}

In conclusion, we have unravelled an infinity of flat band-like, dispersionless eigenstates in the $b=3 N$ class of Sierpinski gasket fractals. The states are localized in clusters of finite size, which are effectively separated from similar clusters in the lattice by atomic sites where the amplitude of the wave function is zero. The topology of the lattice confines the dynamics of the electron in such local clusters for these special sets of energy eigenvalues. The states have been obtained by explicit construction and the inherent self similarity of the lattice is exploited through a real space rescaling of the system. The dispersionless character of the flat band states are confirmed by obtaining the $E$ - $k$ dispersion curves of a periodic array of the approximants of the true gasket, and also from the diverging character of the local density of states at those special energy eigenvalues. The two terminal transport and the magnetic field induced absolutely continuous subbands have been discussed in details. The intricate mixture of complexity, frustration and a deterministic geometry may throw up new challenges in the quantum Hall physics in such fractal geometries. 


\section{Acknowledgments}

AN acknowledges a research fellowship from UGC, India and BP is thankful to DST, India for an INSPIRE fellowship. AC thanks Bibhas Bhattacharyya and Santanu K. Maiti for enlightening discussions.
[1] R. Moessner and A. P. Ramirez, Physics Today, February, p.24 (2006).

[2] H. Kikuchi, Y. Fujii, M. Chiba, S. Mitsudo, T. Idehara, T. Tonegawa, K. Okamoto, T. Sakai, T. Kuwai, and H. Ohta, Phys. Rev. Lett. 94, 227201 (2005).

[3] A. M. S. Macêdo, M. C. dos Santos, M. D. CoutinhoFilho, and C. A. Macêdo, Phys. Rev. Lett. 74, 1851 (1995).

[4] O. Derzhko and J. Richter, Phys. Rev. B 70, 104415 (2004).

[5] O. Derzhko and J. Richter, Phys. Rev. B 72, 094437 (2005).

[6] O. Derzko, J. Richter, and A. Honecker, J. Phys.: Conf. Series 145, 012059 (2009).

[7] O. Derzhko, J. Richter, A. Honecker, M. Maksymenko, and R. Moessner, Phys. Rev. B 81, 014421 (2010).

[8] A. Mielke, J. Phys. A: Math. Gen. 24, L73 (1991); 24, 3311 (1991).

[9] H. Tasaki, Phys. Rev. Lett. 69, 1608 (1992).

[10] A. A. Lopes and R. G. Dias, Phys. Rev. B 84, 085124 (2011).

[11] M. Hyrkäs, V. Apaja, and M. Manninen, Phys. Rev. A 87, 023614 (2013).

[12] A. A. Lopes, B. A. Z. António, and R. G. Dias, Phys. Rev. B 89, 235418 (2014).

[13] S. Flach, D. Leykam, J. D. Bodyfelt, P. Matthies, and A. S. Desyatnikov, Europhys. Lett. 105, 30001 (2014).

[14] D. Leykam, S. Flach, O. B.-Treidel, and A. S. Desyatnikov, Phys. Rev. B 88, 224203 (2013).

[15] M. Vanević, V. M. Stojanović, and M. Kindermann, Phys. Rev. B 80, 045410 (2009).

[16] M. Ezawa, arXiv:1404.5788v1 [cond-mat.mes-hall] (2014).

[17] T. G. Pedersen, C. Flindt, J. Pedersen, N. A. Mortensen, A.-P. Jauho, and K. Pedersen, Phys. Rev. Lett. 100, 136804 (2008).
[18] G.-W. Chern, C.-C. Chien, and M. Di Ventra, Phys. Rev. A 90, 013609 (2014).

[19] G.-B. Jo, J. Guzman, C. K. Thomas, P. Hosur, A. Vishwanath, and D. M. Stamper-Kurn, Phys. Rev. Lett. 108, 045305 (2012).

[20] K. Shiraishi, H. Tamura, and H. Takayanagi, Appl. Phys. Lett. 78, 3702 (2001).

[21] I. Syôzi, Prog. Theo. Phys. 6, 306 (1951).

[22] S. Miyahara, K. Kubo, H. Ono, Y. Shimoumura, and N. Furukawa, J. Phys. Soc. Jpn. 74, 1918 (2005).

[23] E. Domany, S. Alexander, D. Bensimon, and L. P. Kadanoff, Phys. Rev. B 28, 3110 (1983).

[24] R. Rammal, J. Phys. (Paris) 45, 191 (1984); Phys. Rev. B 28, 4871 (1983).

[25] J. R. Banavar, L. P. Kadanoff, and A. M. M. Pruisken, Phys. Rev. B 31, 1388 (1985).

[26] X. R. Wang, Phys. Rev. B 51, 9310 (1995).

[27] J. T. Chalker, T. S. Pickles, and P. Shukla, Phys. Rev. B 82, 104209 (2010).

[28] A. Chakrabarti, J. Phys.: Condens. Matter 810951 (1996).

[29] S. Kirkpatrick and T. P. Eggarter, Phys. Rev. B 6, 3598 (1972).

[30] B. Pal and A. Chakrabarti, Phys. Rev. B 85, 214203 (2012).

[31] A. Nandy, B. Pal, and A. Chakrabarti, Phys. Lett. A 378, 3144 (2014).

[32] T. Stošić, B. Stošić, S. Milošević, and H. E. Stanley, Phys. Rev. E 49, 1009 (1994).

[33] K. Nikolić and A. MacKinnon, Phys. Rev. B 47, 6555 (1993).

[34] H.-F. Cheung, Y. Gefen, E. K. Riedel, and W.-H. Shih, Phys. Rev. B 37, 6050 (1988).

[35] D. R. Hofstadter, Phys. Rev. B 14, 2239 (1976).

[36] Y. Liu and K. A. Chao, Phys. Rev. B 34, 5247 (1986). 